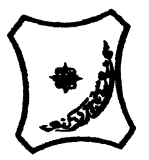

Bayero Journal of Pure and Applied Sciences, 12(1):215 - 220

ISSN 2006 - 6996

\title{
NORMAL TISSUE TOLERANCE DOSE FOR CERVICAL RADIOTHERAPY TO THE NTCP MODEL USING A METHOD OF LEAST SQUARE FIT
}

\author{
Onuh, $E^{1}$. , Ajuji, A. $\mathbf{S}^{2}$. , Rabiu, $\mathbf{N}^{3} .$, Anas, M.S ${ }^{4}$. and Jada, $\mathbf{M}^{5}$. \\ 1,3,4 Department of Physics, Ahmadu Bello University, Zaria. Nigeria. \\ ${ }^{2}$ Centre for Energy Research and Training, Zaria. Nigeria. \\ ${ }^{5}$ National Research Institute for chemical Technology, Zaria. Nigeria. \\ Email: ${ }^{1}$ chesbabe2006@yahoo.com,
}

\begin{abstract}
The purpose of this study is to evaluate the impact of Normal Tissue Complication Probability (NTCP) models on radiation treatment plans. We estimated NTCP parameters for the organs at risk (OARs) for cervical cancer radiotherapy. We pooled individual patient data from fifty patients who were treated with External Beam Radiotherapy technique between March 2012 and November 2013 in a part of Northern Nigeria with the exclusion of patients who had other gynaecological malignancies. Three basic methods were followed during the radiotherapy planning of these patients which included the pre-planning stage, planning radiotherapy treatment stage and treatment delivery. Various tests were carried out on these patients which helped to confirm the diagnosis after which they were treated using the linear accelerator, computed tomography simulator and the treatment planning system. We calculated the normal tissue tolerance doses for partial volumes of the organs using the values of the above said parameters for published data on normal tissue tolerance doses. This article shows a graphical representation of the computed NTCP for left femur, right femur and skin presented and a fairly good correspondence is found between the curves for head of femurs and skin.

Keywords: Normal Tissue Tolerance Dose, Normal Tissue Complication Probability, Method of Least Square Fit, Cervical Radiotherapy
\end{abstract}

\section{INTRODUCTION}

Cervical cancer is a malignant neoplasm arising from cells originating in the cervix uteri. One of the most common symptoms of cervical cancer is an abnormal vaginal bleeding, but in some cases there may be no obvious abnormal vaginal bleeding until the cancer has progressed to an advanced stage (Kumar et al., 2007). Other symptoms of cervical cancer include bleeding after sex (postcoital bleeding), bleeding outside the normal periods, pain and discomfort during sex, an unpleasant smelling vaginal discharge and new bleeding after menopause. Advanced symptoms include constipation, blood, loss of bladder control, bone pain, swelling of one of your legs, changes to your bowel and bladder habits amongst others. Treatment usually consists of surgery in early stages, and chemotherapy and/or radiotherapy in advanced stages of the disease (Tim et al. 2012). In developed countries, the widespread uses of cervical screening programs have dramatically reduced the incidence of invasive cervical cancer. External Beam Radiation Therapy (EBRT) is a type of radiation therapy that directs a beam of radiation from outside the body at cancerous tissues within the body using a special $\mathrm{X}$-ray machine called a linear accelerator. This machine allows radiation to be delivered from any angle and shapes radiation beams to the contour of the tumour (ACS, 2014). External beam radiation treatments include the three dimensional conformal radiation therapy (3D Conformal RT) and the intensity modulated radiation therapy (IMRT) (ACS, 2014). Normal Tissue Complication Probability is the probability that a given dose of radiation will cause an organ or structure to experience complications considering the specific biological cells of the organ or structure.

Therefore, this works aims to look at the tolerance doses for organs at risk for cervical radiotherapy using the N.T.C.P model. 
Special Conference Edition, November, 2019 MATERIALS AND METHODS

A random selection of cervical cancer patients, who were treated at the Usman Danfodio University Teaching Hospital, Sokoto (UDUTH) in the period between March 2012 and November 2013, were included in the study. The population sample size was a total of fifty patients treated in a supine position on one of the linear accelerator (LINACS). The machine has Multiple energies between the range of 6 and 15MV for photons and $2-18 \mathrm{MeV}$ for electrons. All patients were simulated in the supine position on a CT-Simulator. Five skin marks, one at the centre of the anterior field and two anti-rotational tattoos on both the lateral sides of the patient were marked and simulation films were taken. Treatment fields of Anteriorposterior (AP) and Posterior-anterior (PA) fields or a four field technique (AP, PA, right and left lateral fields) were planned with $2 \mathrm{~cm}$ margins defined by the clinician from bony anatomy. Patients were treated with a $100 \mathrm{~cm}$ Source to Axis Distance (SAD) isocentric technique. The treatment couch digital readout of this verified patient position was taken as the intended or planned setup position in the study. Using LANTIS $^{\text {TM }}$, the digital daily treatment couch absolute positions of each patient in terms of the $X, Y$, and $Z$ position were recorded. These corresponded to the medio-lateral, superoinferio and anterior-posterior position of the patient respectively.

Using the beam set up parameters for patients, the tumour volume for patients with threedimensional plans was estimated from the dimensions of the set field size used to treat the particular patient. For example, for a patient treated using four isocentric fields namely a 13 $\mathrm{cm} \times 15 \mathrm{~cm}$ anterior posterior field and $8.6 \mathrm{~cm} \times$ $15 \mathrm{~cm}$ left and right lateral oblique field, the tumour volume was determined from the geometric boundaries of the set treatment fields i.e. $13 \mathrm{~cm} \times 15 \mathrm{~cm} \times 8.6 \mathrm{~cm}$. This is an overestimation of the "tumour volume" because the treated volume included normal tissue around the tumour. As a result of this method used, the tumour volume of the threedimensional plans was in most cases very large and in order to solve this problem, the tumour volume was scaled down to a smaller volume and this was done by dividing the tumour volume by a constant reference volume. The reference volume was taken to be $9.9 \mathrm{~cm} 3$. This value was gotten by taking $10 \%$ of the actual volume (Table 3 ) of the irradiated organ for each patient since the reference volume must always be a constant volume.
Sokoto state was created as a state in 1976. In terms of density, it is ranked 16 out of the 36 states. Sokoto state has been ruled by governors, most are ex-military officers, who succeeded each another at short intervals. It is located in the extreme northwest of Nigeria, near to the confluence of the Sokoto River and the Rima River. As of 2006 census, it had an estimated population of more than 4.2 million. It is the modern day capital of Sokoto State (and its predecessor, the Northwestern State). Over eighty percent $(80 \%)$ of the inhabitants of Sokoto practice one form of agriculture or the other. They produce such crops as millet, guinea corn, maize, rice, potatoes, cassava, groundnuts and beans for subsistence and produce wheat, cotton and vegetable for cash. Local crafts such as blacksmithing, weaving, dyeing, carving and leather works also play an important role in the economic life of the people. Sokoto is also one of the fish producing areas of the country (www.onlinenigeria.com/map).

\section{NTCP Calculations With Respect To The} Linear Quadratic Model

All the 50 patients who were eligible for this study had a three dimensional treatment plan. The Normal tissue complication probability equation used in this study is given as follow; $\operatorname{NTCP}(D, v)=\exp \left[-N_{0} V^{-K} \exp (-\alpha G D)\right]$

(1) (Emami et al., 1991)

where; $\operatorname{NTCP}(D, v)=$ The Normal Tissue Complication Probability with respect to the normal tissue dose delivered per fraction and volume of the normal tissue.

$\mathrm{N}_{0}$ and $\mathrm{k}$ are the tissue / organ specific, nonnegative adjustable parameters.

$\mathrm{V}$ is the uniformly irradiated partial volume of the tissue / organ (This research work made use of whole volume)

$\mathrm{D}$ is the normal tissue dose delivered with dose per fraction.

\section{Fitting Parameters}

The least square fitting was done using the equation given as

follows;

$f(x)=\exp [-a \times \exp \{-b x\}]$

Where $a$ and $b$ are the fitting parameters. Comparing equation (2) with equation (1) k was calculated from the fitting equation as; $a=N V^{-K}$

$K=\frac{(\operatorname{LnN}-\operatorname{Lna})}{\ln V}$

where $a$ is the fitting parameter. 
Special Conference Edition, November, 2019

The calculated values of $\mathrm{k}$ and $\mathrm{aG}$ are listed in table 1 along with those of Emami and coworkers. Due to the unavailability of adequate data, no attempt was made to correlate the NTCP with a reference volume. To solve this problem and to simplify the calculation, the organs were assumed to have the same reference volume. A $\mathrm{C}++$ program code was written to help in the plotting computation and the Gnu plot plotting software was used for both the plots and fitting.

\section{RESULTS AND DISCUSSION}

Using equation 1 , the values of the constants given in Table 1, the volume of irradiated organ given in Table 3 and the values of the doses given to the organs in Table 2, the NTCP values for the different organs (left Femur, right femur and skin) were calculated and presented in Table 3. The values of the Normal Tissue Complication Probability for the various organs were found to be in the range of 0 and 1 .

Fig 1 shows a curve of NTCP against left femoral dose. It is seen from the graph that as the dose to the left femur increases, the probability of complication also increases. The threshold values for whole volume and $1 / 3$ partial volume is given as $5 \mathrm{~Gy}$ and $18 \mathrm{~Gy}$ respectively while the threshold value for $3 / 3$ volume falls within the negative portion of the graph. At a certain dose say about $17.5 \mathrm{~Gy}$, it will be observed that no complication arises for $1 / 3$ partial volume but a large portion of the curve gets complicated for the whole volume and $3 / 3$ partial volume. This study therefore, shows that for left femur, it is safer to administer doses at smaller volumes say $1 / 3$ of the whole volume because at smaller volumes, little or no damage is done to the left femur. At whole volume, even though the probability of complication is about $0.1(10 \%)$ more portion of the left femur is damaged. For the $3 / 3$ volume it is seen that the damage done to the left femur rises to about $0.5(50 \%)$ with a greater portion of the femur being damaged. The value of the fitting parameters $\alpha$ and $b(a G)$ have been calculated to be approximately 6.7145 and 0.0174 respectively.

Fig 2 is a curve of NTCP against right femoral dose. Also, this curve does not show the sigmoid shape within the range of values and simply reduces the exponential shape. This is due to the fact that from Table 1, the right femur has a higher value of $a G$ (the higher the value of $a G$, the less steep the curve from the linear quadratic model) than that of the left femur. The value of the NTCP for the $1 / 3$ volume and whole volume begins to appreciate at a dose of 12Gy and $2 \mathrm{~Gy}$ respectively. At a dose of $12 \mathrm{~Gy}$, it will be observed that the probability of complication to the whole volume and $3 / 3$ partial volume is $14 \%$ and $50 \%$ respectively with no complication for $1 / 3$ partial volume. Therefore, this research work brings out the fact that for right femur, it is better to irradiate smaller volumes $(1 / 3)$ at a given dose of $12 \mathrm{~Gy}$ as this dose gives no complication. For whole volumes, a dose of about 2Gy gives no complication while for $3 / 3$ volume the threshold dose falls within the negative portion of the curve. Again, it is observed that a fairly large portion of the whole volume becomes damaged. This is due to the large value of $k$. The higher the value of $k$, the larger the volume of the organ that becomes damaged. The volume effect is largely dependent on the k-parameter (equation 1). The $\mathrm{k}$ value is approximately 2.4 which is quite high. The fitting parameters $a$ and $b$ were calculated to be 5.601 and 0.1026 respectively.

Table 1: Values of the fitted parameters for this work and that of Emami. The symbol $E$ stands for error.

\begin{tabular}{lcccccccc}
\hline Organ & \multicolumn{3}{l}{ This Work } & \multicolumn{4}{c}{ Emami's Work (Emami et al, 1991) } \\
& $\mathrm{k}$ & $\alpha G$ & $\mathrm{~N}$ & $\Delta K$ & $\Delta \alpha G(E)$ & $\mathrm{k}$ & $\alpha G$ & $\mathrm{~N}$ \\
\hline Right femur & 2.3641 & 0.1037 & 1045.23 & 2.3641 & 0.0089 & 0 & 0.1126 & 1045.23 \\
Left femur & 2.2685 & 0.0735 & 1045.23 & 2.2685 & 0.0391 & 0 & 0.1126 & 1045.23 \\
Skin & 1.6420 & 0.0882 & 351.42 & 1.0553 & 0.0004 & 0.5867 & 0.0886 & 351.42 \\
\hline
\end{tabular}

Table 2: Some values for Doses (Gy) for the various Organs of interest

\begin{tabular}{lcc}
\hline Head of Femur dose (Gy) & Skin Dose (Gy) \\
\hline Left & Right & \\
18 & 18 & 16 \\
17 & 17 & 15 \\
1 & 1 & 14 \\
29 & 29 & 17 \\
\hline
\end{tabular}


Special Conference Edition, November, 2019

Table 3: Some values for Volume $(\mathrm{cm} 3)$ of irradiated Organs and values of NTCP for the different Organs

\begin{tabular}{llll}
\hline Volume of Irradiated Tissue $\left(\mathrm{cm}^{3}\right)$ & \multicolumn{3}{c}{ NTCP values for the different organs } \\
& \multicolumn{2}{c}{ Head of femurs } & skin \\
& Left & Right & \\
\hline 1000.00 & 0.1616 & 0.3819 & 0.1084 \\
1320.00 & 0.1407 & 0.0029 & 0.0882 \\
1300.00 & 0.0017 & 0.7793 & 0.0705 \\
0893.04 & 0.4427 & 0.6455 & 0.1309 \\
2116.80 & 0.3089 & 0.0000 & 0.1084 \\
0000.00 & 0.0000 & 0.7793 & 0.0000 \\
\hline
\end{tabular}

Fig 3 is a curve of NTCP against skin dose. In this curve, it is observed that the values of NTCP begins to appreciate at a dose of about 7Gy for whole volume, $18 \mathrm{~Gy}$ for $1 / 3$ partial volume and below 0 for $3 / 3$ partial volume. These threshold values simply mean that for whole volume, one starts to observe complications when a dose of about 7Gy is administered. While for $1 / 3$ partial volume complication begins at $18 \mathrm{~Gy}$. This study therefore shows that irradiation of the whole volume and $1 / 3$ partial volume of the skin at a maximum dose of 7Gy and 20Gy is safer for the patients. If doses greater than these values (7Gy and 20Gy) are administered to the whole and $1 / 3$ partial volumes of the skin, complications will be seen. Irradiation of the skin at $3 / 3$ of the whole volume is quite unreasonable as doses less than zero cannot be administered to the patients therefore, the decision of the clinical oncologist may be required. The whole volume curve would have exhibited the sigmoid shape if the dose values were extended beyond 25Gy. Fig 3 is a steep curve this may be due to the fact that the value of $a G$ which is a parameter that determines the steepness of the curve is low when compared to that of Right femur. The value of the fitting parameters $a$ and $b$ have been calculated to be approximately 9.6614 and 0.0909 respectively.

Table 1 shows the values of the constants (aG and $\mathrm{K}$ ) calculated for this research and the values used. It can be seen that the constant values (k) for Right femur, left femur and Skin is high when compared with the values gotten by the author, Emami et al (1991). Also, the value of the constants (aG) for all the organs used in this work shows a close range of value when compared with the work done by the author. These differences in values could be as a result of the fact that this research work made use of whole volume of the organ irradiated when compared with that of the author who used partial volume. Another reason could be as a result of the fact that the treatment planning system used by the author was a 2D treatment planning system whereas this work made use of a 3D-treatment planning system.

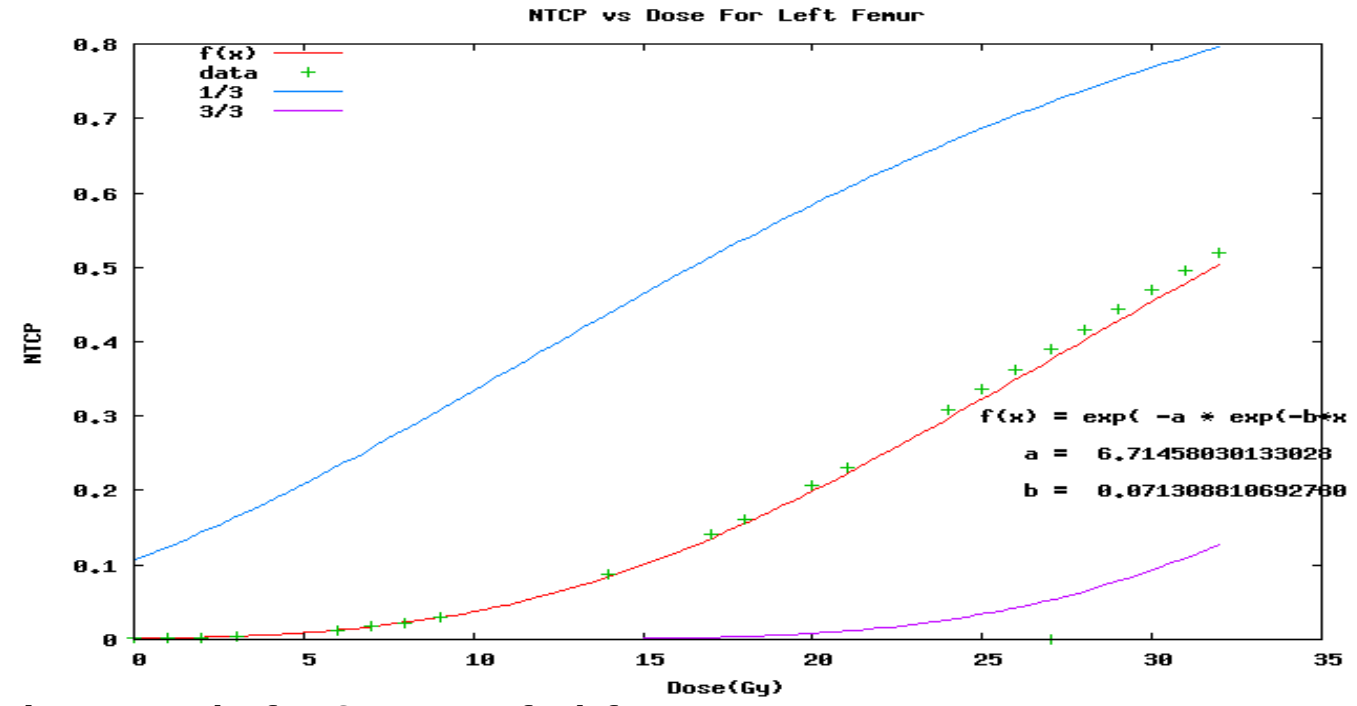

Fig 1; A graph of NTCP vs Dose for left Femur 


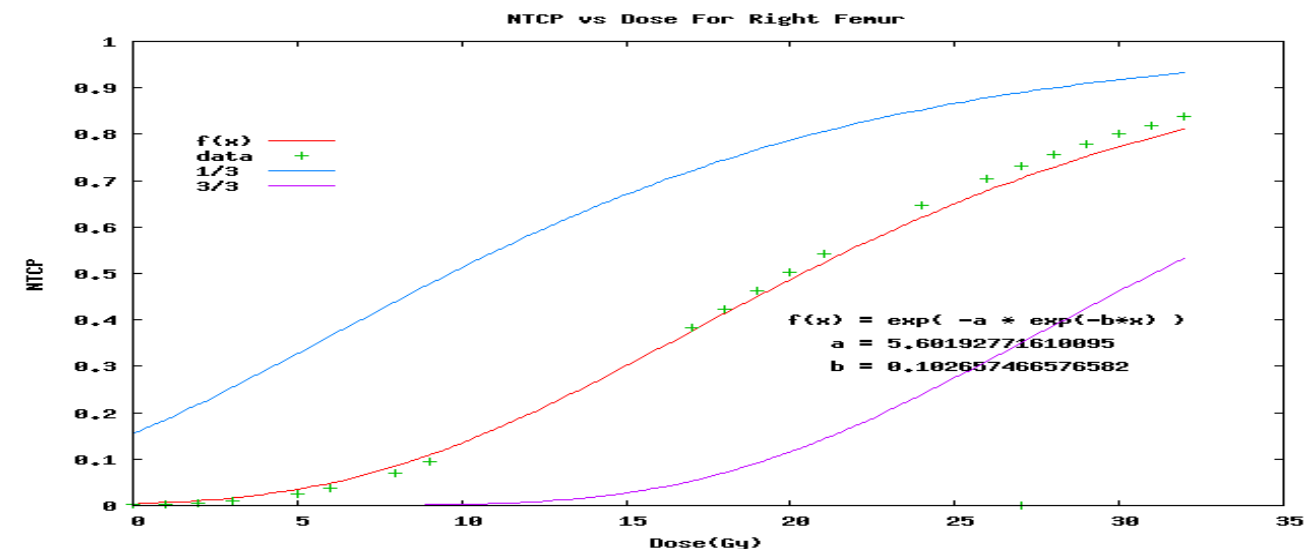

Fig 2; A graph of NTCP vs Dose for right Femur

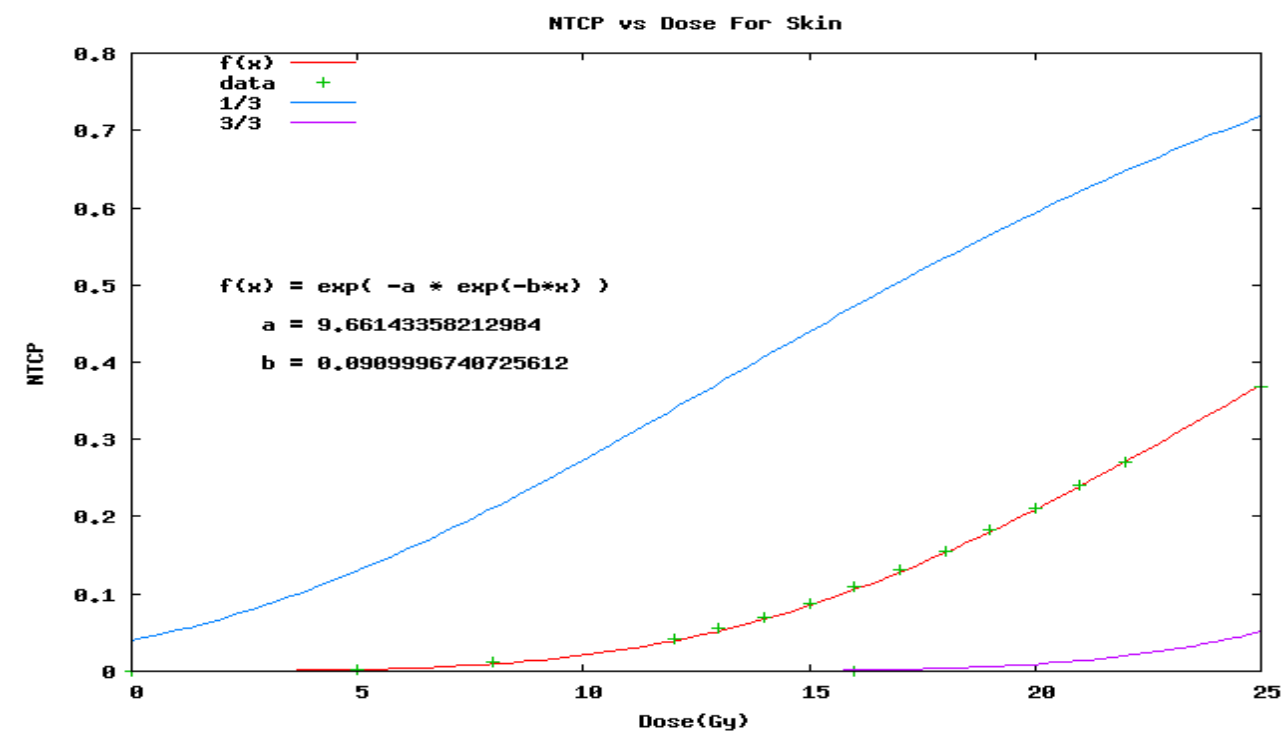

Fig 3; A graph of NTCP vs Dose for right Femur

\section{CONCLUSION}

The various stages of cervical cancer in the sampled patients were from stages I - IV. Different fractionation schedules were used for different patients and various doses of radiation were also administered to the different patient depending on the stage of cervical cancer.

From the various graphs, it is seen that a fairly good result has been found between the curves. For each figure, it was generally observed that a good relationship exists between the N.T.C.P value and the organ (right and left femurs and skin) doses given. As the doses increases for all the organs, the probability of complication gets larger for these ranges $(0 \%-100 \%)$. This observed result is in agreement with what Emami et al. (1991) have reported. For all the organs, it was observed that the smaller the volume of the organ, the lesser the complication to these organs (head of femurs and skin). For the right femur and skin, it was observed that the threshold value for $3 / 3$ partial volumes of these organs are negative and since doses below 0Gy (beyond therapeutic range) cannot be administered to patients, the clinical judgment of the treating physician prevails in the treatment decision. The fitted values of $k$ and aG obtained in this work shows significant discrepancies when compared with the work done by Emami et al. (1991). This may be due to the fact that in the work done by Emami et al, the conventional 2D-Treatment planning system was used unlike the 3D-treatment planning system which was used for this research work. Secondly, doses exceeding 50Gy were not administered to the patients under this study at UDUTH whereas in the case of Emami et al doses as high as $125 \mathrm{~Gy}$ were administered to the patients. Values of $\mathrm{k}$ for the various organs considered in this work indicate that organs which have high values of $k>1$, have a higher volume dependency than organs which have lower values of $k<1$. 


\section{REFERENCES}

Andrews, J.R., Amer. J. Roentgen., Moody J.M. (1956). The dose-time relationship in radiotherapy. 75:590-596

Burman C., Kutcher G. J., Emami B., Goiten M. (1991). Fitting of normal tissue tolerance data to an analytic function. International Journal on Radiation Oncology BiolPhysic, 21: $123-135$.

Emami B., Graham M.V. (1997). Lung. In : Principles and Practice of Radiation Oncology, Parez CA \& Brady LW, eds, 3 edition, Philadelphia, pp. 1181-1220.
Emami B., Lyman J., Brown A., Coia L., Goiten M., Munzenride J. E., Shank B., Solin L. J., Wesson M. (1991). Tolerance of normal tissue to therapeutic radiation. International Journal on Radiation Oncology BiolPhysic. 21: $109-122$

Fowler J.F. (1989). The linear-quadratic formula and progress in fractionated radiotherapy. Br J Radiol, 62(740): 67994.

Kallman P., Agren A., Brahme A. (1992). Tumor and normal tissue responses to fractionated non-uniform dose delivery. International. Journal on Radiation Biol. Physic. 62: 249-262 